\title{
Antiperiodic Boundary Value Problems for Finite Dimensional Differential Systems
}

\author{
Y. Q. Chen, ${ }^{1}$ D. O'Regan, $^{2}$ F. L. Wang, ${ }^{1}$ and S. L. Zhou ${ }^{1}$ \\ ${ }^{1}$ Faculty of Applied Mathematics, Guangdong University of Technology, Guangzhou, \\ Guangdong 510006, China \\ ${ }^{2}$ Department of Mathematics, National University of Ireland, Galway, Ireland
}

Correspondence should be addressed to D. O’Regan, donal.oregan@nuigalway.ie

Received 16 March 2009; Accepted 28 May 2009

Recommended by Juan J. Nieto

We study antiperiodic boundary value problems for semilinear differential and impulsive differential equations in finite dimensional spaces. Several new existence results are obtained.

Copyright (C) 2009 Y. Q. Chen et al. This is an open access article distributed under the Creative Commons Attribution License, which permits unrestricted use, distribution, and reproduction in any medium, provided the original work is properly cited.

\section{Introduction}

The study of antiperiodic solutions for nonlinear evolution equations is closely related to the study of periodic solutions, and it was initiated by Okochi [1]. During the past twenty years, antiperiodic problems have been extensively studied by many authors, see [1-31] and the references therein. For example, antiperiodic trigonometric polynomials are important in the study of interpolation problems [32,33], and antiperiodic wavelets are discussed in [34]. Moreover, antiperiodic boundary conditions appear in physics in a variety of situations, see [35-40]. In Section 2 we consider the antiperiodic problem

$$
\begin{gathered}
u^{\prime}(t)=A u(t)+f(t, u(t)), \quad t \in R, \\
u(t)=-u(t+T), \quad t \in R,
\end{gathered}
$$

where $A$ is an $n \times n$ matrix, $f: R \times R^{n} \rightarrow R^{n}$ is continuous, and $f(t+T, x)=-f(t, x)$ for all $(t, x) \in R \times R^{n}$. Under certain conditions on the nondiagonal elements of $A$ and $f$ we prove an existence result for (E 1.1). In Section 3 we consider the antiperiodic boundary value problem

$$
\begin{gathered}
u^{\prime}(t)=G u(t)+f(t, u(t)), \quad \text { a.e. } t \in J=[0, T], t \neq t_{k}, \\
u(0)=-u(T), \\
\Delta u\left(t_{k}\right)=I_{k}\left(u\left(t_{k}\right)\right), \quad k=1,2, \ldots, p,
\end{gathered}
$$


where $G: R^{n} \rightarrow R^{n}$ is a function satisfying $G 0=0$, and $f: J \times R^{n} \rightarrow R^{n}$ is a Caratheodory function, $\Delta u\left(t_{k}\right)=u\left(t_{k}^{+}\right)-u\left(t_{k}^{-}\right)$, and $I_{k} \in C\left(R^{n}, R^{n}\right)$. Under certain conditions on $G, f$, and $I_{k}(u)$ for $k=1,2, \ldots, p$, we prove an existence result for (E 1.2).

\section{Antiperiodic Problem for Differential Equations in $R^{n}$}

Let $|\cdot|$ be the norm in $R^{n}$. In this section we study

$$
\begin{gathered}
u^{\prime}(t)=A u(t)+f(t, u(t)), \quad t \in R, \\
u(t)=-u(t+T) .
\end{gathered}
$$

First, we have the following result.

Theorem 2.1. Let $A=\left(a_{i j}\right)$ be an $n \times n$ matrix, where $a_{i j}$ is the element of $A$ in the ith row and $j$ th column, $f: R \rightarrow R^{n}$ is continuous and $f(t+T)=-f(t)$ for $t \in R$. Suppose $(T / 2) \Sigma_{1 \leq i<j \leq n}\left|a_{i j}-a_{j i}\right|<$ 1. Then the equation

$$
\begin{gathered}
u^{\prime}(t)=A u(t)+f(t), \quad t \in R \\
u(t)=-u(t+T), \quad t \in R
\end{gathered}
$$

has a unique solution.

Proof. Put $W_{a}=\left\{v(\cdot) \in C\left(R ; R^{n}\right): v(t)=-v(t+T)\right\}$. Then $W_{a}$ is a Banach space under the norm $|v(\cdot)|_{\infty}=\max _{t \in[0, T]}|v(t)|$. For each $v(\cdot) \in W_{a}$, consider the following equation:

$$
\begin{gathered}
u^{\prime}(t)=A v(t)+f(t), \quad t \in R, \\
u(t)=-u(t+T), \quad t \in R .
\end{gathered}
$$

It is easy to see that $u(t)=-(1 / 2) \int_{0}^{T}[A v(s)+f(s)] d s+\int_{0}^{t}[A v(s)+f(s)] d s$ is the unique solution of (E 2.3).

We define a mapping $K: W_{a} \rightarrow W_{a}$ as follows:

$$
\text { for any } v(\cdot) \in W_{a}, \quad K v(\cdot)=u(\cdot), \quad u(\cdot) \text { is the solution of (E 2.3). }
$$

First we prove that $K$ is a continuous compact mapping. Now assume $v_{n}(\cdot) \in W_{a}, n=1,2, \ldots$, and $v_{n}(\cdot) \rightarrow v(\cdot) \in W_{a}$. Then $\left|A v_{n}(\cdot)-A v(\cdot)\right|_{\infty} \rightarrow 0$ as $n \rightarrow \infty$. This immediately implies that $\int_{0}^{T}\left|\left(K v_{n}(t)\right)^{\prime}-(K v(t))^{\prime}\right|^{2} d t \rightarrow 0$ as $n \rightarrow \infty$.

We have $K v_{n}(t)-K v(t)=(1 / 2)\left\{\int_{0}^{t}\left[\left(K v_{n}(s)\right)^{\prime}-(K v(s))^{\prime}\right] d s-\int_{t}^{T}\left[\left(K v_{n}(s)\right)^{\prime}-\right.\right.$ $\left.\left.\left.(K v(s))^{\prime}\right] d s\right]\right\}$, and so $K v_{n}(\cdot) \rightarrow K v(\cdot)$ in $W_{a}$.

Now since $(K v(t))^{\prime}=A v(t)+f(t), t \in R$, it is easy to see that

$$
\left(\int_{0}^{T}\left|(K v(t))^{\prime}\right|^{2} d t\right)^{1 / 2} \leq \sqrt{T}|A v(\cdot)|_{\infty}+\left(\int_{0}^{T}|f(t)|^{2} d t\right)^{1 / 2} .
$$


Thus $K$ maps a bounded subset of $W_{a}$ to a bounded equicontinuous subset in $W_{a}$, therefore $K$ is completely continuous.

Next take $r_{0}>\left(1-(T / 2) \Sigma_{1 \leq i<j \leq n}\left|a_{i j}-a_{j i}\right|\right)^{-1}(\sqrt{T} / 2)\left(\int_{0}^{T}|f(t)|^{2} d t\right)^{1 / 2}$. We show that $K v(\cdot) \neq \lambda v(\cdot)$ for all $\lambda \geq 1$, and $|v(\cdot)|_{\infty}=r_{0}$. If this is not true, there exist $\lambda_{0} \geq 1, w(\cdot) \in W_{a}$ with $|w(\cdot)|_{\infty}=r_{0}$ such that $K w(\cdot)=\lambda_{0} w(\cdot)$, that is, $w(t)=-w(t+T), t \in R$ and

$$
\lambda_{0} w^{\prime}(t)=A w(t)+f(t), \quad t \in R
$$

Multiply (2.3) by $w^{\prime}(t)$ (i.e., take inner product) and integrate over [0,T], and notice that $\int_{0}^{T} w_{i}(t) w_{j}^{\prime}(t) d t=-\int_{0}^{T} w_{i}^{\prime}(t) w_{j}(t) d t$ to get

$$
\lambda_{0} \int_{0}^{T}\left|w^{\prime}(t)\right|^{2} d t \leq \Sigma_{1 \leq i<j \leq n}\left|a_{i j}-a_{j i}\right| \int_{0}^{T}\left|w_{i}(t) w_{j}^{\prime}(t)\right| d t+\left(\int_{0}^{T}|f(t)|^{2} d t\right)^{1 / 2}\left(\int_{0}^{T}\left|w^{\prime}(t)\right|^{2} d t\right)^{1 / 2},
$$

where $w(t)=\left(w_{i}(t)\right), i=1,2, \ldots, n$. Notice that $w(t)=(1 / 2)\left[\int_{0}^{t} w^{\prime}(s) d s-\int_{t}^{T} w^{\prime}(s)\right] d s$, so we have

$$
|w(\cdot)|_{\infty} \leq \frac{\sqrt{T}}{2}\left(\int_{0}^{T}\left|w^{\prime}(t)\right|^{2} d t\right)^{1 / 2}
$$

From (2.4), (2.5), we have

$$
\lambda_{0}\left(\int_{0}^{T}\left|w^{\prime}(t)\right|^{2} d t\right)^{1 / 2} \leq \sqrt{T} \Sigma_{1 \leq i<j \leq n}\left|a_{i j}-a_{j i}\right||w(\cdot)|_{\infty}+\left(\int_{0}^{T}|f(t)|^{2} d t\right)^{1 / 2} .
$$

This with (2.5) gives

$$
\lambda_{0}|w(\cdot)|_{\infty} \leq \frac{T}{2} \Sigma_{1 \leq i<j \leq n}\left|a_{i j}-a_{j i}\right||w(\cdot)|_{\infty}+\frac{\sqrt{T}}{2}\left(\int_{0}^{T}|f(t)|^{2} d t\right)^{1 / 2} .
$$

As a result

$$
|w(\cdot)|_{\infty} \leq\left(1-\frac{T}{2} \Sigma_{1 \leq i<j \leq n}\left|a_{i j}-a_{j i}\right|\right)^{-1} \frac{\sqrt{T}}{2}\left(\int_{0}^{T}|f(t)|^{2} d t\right)^{1 / 2}
$$

which contradicts $|w(\cdot)|_{\infty}=r_{0}$.

Thus the Leray-Schauder degree $\operatorname{deg}\left(I-K, B\left(0, r_{0}\right), 0\right)=1$, where $B\left(0, r_{0}\right)$ is the open ball centered at 0 with radius $r_{0}$ in $C_{a}$. Consequently, $K$ has a fixed point in $B\left(0, r_{0}\right)$, that is, (E 2.2) has a solution. For the uniqueness, if $u(\cdot), v(\cdot)$ are two solutions of (E 2.2), set $w(t)=$ $u(t)-v(t)$, then $w^{\prime}(t)=A w(t)$, and $w(t)=-w(t+T)$, for $t \in R$. Following the obvious 
strategy above (see the clear adjustment of (2.8)) gives $|w(\cdot)|_{\infty}=0$. Thus the solution of (E 2.2) is unique.

From Theorem 2.1 we have immediately the following result.

Corollary 2.2. Let $A=\left(a_{i j}\right)$ be an $n \times n$ symmetric matrix, $f: R \rightarrow R^{n}$ is continuous and $f(t+T)=-f(t)$ for $t \in R$. Then

$$
\begin{gathered}
u^{\prime}(t)=A u(t)+f(t), \quad t \in R \\
u(t)=-u(t+T), \quad t \in R
\end{gathered}
$$

has a unique solution.

Using a proof similar to Theorem 2.1, we have the following result.

Theorem 2.3. Let $A=\left(a_{i j}\right)$ be an $n \times n$ matrix, $G: R^{n} \rightarrow R^{n}$ is an even continuously differentiable function, and $f(t, u): R \times R^{n} \rightarrow R^{n}$ is continuous and $f(t+T, u)=-f(t, u)$ for $(t, u) \in R \times R^{n}$. Suppose the following conditions are satisfied:

(1) $|f(t, x)| \leq M|x|+g(t)$, for a.e. $(t, x) \in R \times R^{n}$, where $M>0$ is a constant, and $g(\cdot) \in$ $L^{2}(0, T)$;

(2) $(T / 2)\left[\Sigma_{1 \leq i<j \leq n}\left|a_{i j}-a_{j i}\right|+M\right]<1$.

Then

$$
\begin{gathered}
u^{\prime}(t)=A u(t)+\partial G u(t)+f(t, u(t)), \quad t \in R, \\
u(t)=-u(t+T), \quad t \in R
\end{gathered}
$$

has a solution.

Remark 2.4. Equation (E 2.5) was studied by Haraux [18] and Chen et al. [14] in the case $A=0$, and also by Chen [12] with different assumptions on $f$ and $A$.

\section{Antiperiodic Boundary Value Problem for Impulsive ODE}

In this section, we prove an existence result for the equation

$$
\begin{gathered}
u^{\prime}(t)=G u(t)+f(t, u(t)), \quad \text { a.e. } t \in J=[0, T], t \neq t_{k}, \\
u(0)=-u(T), \\
\Delta u\left(t_{k}\right)=I_{k}\left(u\left(t_{k}\right)\right), \quad k=1,2, \ldots, p,
\end{gathered}
$$

where $G: R^{n} \rightarrow R^{n}$ is a Lipschitz function. We first introduce some notations. Let $J=[0, T]$, and $0=t_{0}<t_{1}<\cdots<t_{p}<t_{p+1}=T . P C(J)=\left\{u: J \rightarrow R^{n}, u_{\left(t_{k}, t_{k+1}\right]} \in\right.$ $C\left(\left(t_{k}, t_{k+1}\right], R^{n}\right), k=0,1, \ldots, p, u\left(t_{k}^{-}\right)$exist for $k=1,2, \ldots, p$, and $\left.u\left(0^{+}\right)=u(0)\right\}$, and $P W^{1,2}(J)=\left\{u \in P C(J): u_{\left(t_{k}, t_{k+1}\right)} \in W^{1,2}\left(\left(t_{k}, t_{k+1}\right), R^{n}\right), k=1, \ldots, p\right\}$. It is clear that $P C(J)$ 
and $P W^{1,2}(J)$ are Banach spaces with the respective norm $\|u\|_{P C(J)}=\sup \{|u(t)|, t \in J\}$, and $\|u\|_{P W^{1,2}(J)}=\sum_{k=0}^{p}\left\|u_{k}\right\|_{W^{1,2}\left(t_{k}, t_{k+1}\right)}$, where $u_{k}:\left(t_{k}, t_{k+1}\right] \rightarrow R$ is defined by $u_{k}(t)=u(t)$ for $t \in\left(t_{k}, t_{k+1}\right], k=0,1, \ldots, p$.

We say a function $u$ is a solution of (E 3.1) if $u \in P W^{1,2}(J)$ and $u$ satisfies (E 3.1).

We first prove the following result.

Lemma 3.1. Let $I_{i}: R^{n} \rightarrow R^{n}$ be continuous functions for $i=1,2, \ldots, p$, and $\sum_{k=1}^{p}\left|I_{k}\left(x_{k}\right)\right| \leq$ $\alpha\left\{\max _{1 \leq k \leq p}\left|x_{k}\right|\right\}+\delta$ for all $x_{k} \in R^{n}, k=1,2, \ldots, p$, where $\alpha, \delta>0$ are constants, and $\alpha<2$. Suppose $u \in P W^{1,2}(J)$ with $u(0)=-u(T)$, and $\Delta u\left(t_{i}\right)=I_{i}\left(u\left(t_{i}\right)\right)$, for $i=1,2, \ldots, p$. Then

$$
\|u\|_{P C(J)} \leq\left(1-\frac{1}{2} \alpha\right)^{-1}\left[\frac{1}{2} \delta+\frac{\sqrt{T}}{2}\left(\int_{0}^{T}\left|u^{\prime}(s)\right|^{2} d s\right)^{1 / 2}\right] .
$$

Proof. By assumption, we have $u(t)=u(0)+\int_{0}^{t} u^{\prime}(s) d s$ for $t \in\left[0, t_{1}\right)$, and

$$
u(t)=u(0)+\sum_{i=1}^{k} I_{i}\left(u\left(t_{i}\right)\right)+\int_{0}^{t} u^{\prime}(s) d s
$$

for $t \in\left[t_{k}, t_{k+1}\right), k=1,2, \ldots, p$. Since $u(0)=-u(T)$, it follows that $u(t)=-(1 / 2)\left[\sum_{i=1}^{p} I_{i}\left(u\left(t_{i}\right)\right)+\right.$ $\left.\int_{0}^{T} u^{\prime}(s) d s\right]+\int_{0}^{t} u^{\prime}(s) d s$ for $t \in\left[0, t_{1}\right)$, and

$$
u(t)=-\frac{1}{2}\left[\sum_{i=1}^{p} I_{i}\left(u\left(t_{i}\right)\right)+\int_{0}^{T} u^{\prime}(s) d s\right]+\sum_{i=1}^{k} I_{i}\left(u\left(t_{i}\right)\right)+\int_{0}^{t} u^{\prime}(s) d s
$$

for $t \in\left[t_{k}, t_{k+1}\right), k=1,2, \ldots, p$. Hence we have

$$
\|u\|_{P C(J)} \leq \frac{1}{2}\left[\alpha\|u\|_{P C(J)}+\delta\right]+\frac{\sqrt{T}}{2}\left(\int_{0}^{T}\left|u^{\prime}(s)\right|^{2} d s\right)^{1 / 2} .
$$

Thus

$$
\|u\|_{P C(J)} \leq\left(1-\frac{1}{2} \alpha\right)^{-1}\left[\frac{1}{2} \delta+\frac{\sqrt{T}}{2}\left(\int_{0}^{T}\left|u^{\prime}(s)\right|^{2} d s\right)^{1 / 2}\right] .
$$

Theorem 3.2. Let $G: R^{n} \rightarrow R^{n}$ be a function satisfying $G 0=0$, and $f:[0, T] \rightarrow R^{n}$ such that $f(\cdot) \in L^{2}([0, T])$, and let $I_{k}: R^{n} \rightarrow R^{n}$ be continuous functions for $k=1,2, \ldots, p$. Suppose the following conditions are satisfied:

(1) $|G u-G v| \leq L|u-v|$ for all $u, v \in R^{n}$, and $L>0$ is a constant;

(2) $\Sigma_{k=1}^{p}\left|I_{k}\left(x_{k}\right)\right| \leq \gamma\left\{\max _{1 \leq k \leq p}\left|x_{k}\right|\right\}+\delta$ for all $x_{k} \in R^{n}, k=1,2, \ldots, p$, where $\gamma, \delta>0$ are constants;

(3) $\gamma+T L<2$. 
Then the problem

$$
\begin{gathered}
u^{\prime}(t)=G u(t)+f(t), \quad \text { a.e. } t \in J=[0, T], t \neq t_{k}, \\
u(0)=-u(T), \\
\Delta u\left(t_{k}\right)=I_{k}\left(u\left(t_{k}\right)\right), \quad k=1,2, \ldots, p
\end{gathered}
$$

has a solution.

Proof. For each $v \in P C(J)$, consider the problem

$$
\begin{gathered}
u^{\prime}(t)=G v(t)+f(t) \quad \text { a.e. } t \in J=[0, T], t \neq t_{k} \\
u(0)=-u(T) \\
\Delta u\left(t_{k}\right)=I_{k}\left(v\left(t_{k}\right)\right), \quad k=1,2, \ldots, p .
\end{gathered}
$$

One can easily show that the solution $u$ of (E 3.3) is given by the following:

$$
\begin{aligned}
u(t)= & -\frac{1}{2}\left[\sum_{i=1}^{p} I_{i}\left(v\left(t_{i}\right)\right)+\int_{0}^{T}(G v(s)+f(s)) d s\right] \\
& +\int_{0}^{t}(G(v(s))+f(s)) d s, \quad \text { for } t \in\left[0, t_{1}\right), \\
u(t)= & -\frac{1}{2}\left[\Sigma_{i=1}^{p} I_{i}\left(v\left(t_{i}\right)\right)+\int_{0}^{T}(G v(s)+f(s)) d s\right]+\sum_{i=1}^{k} I_{i}\left(v\left(t_{i}\right)\right) \\
& +\int_{0}^{t}(G v(s)+f(s)) d s,
\end{aligned}
$$

for $t \in\left[t_{k}, t_{k+1}\right), k=1, \ldots, p$.

Obviously, the solution of (E 3.3) is unique. Now we define $K: P C(J) \rightarrow P W^{1,2}(J) \subset$ $P C(J)$ by $u=K v$. We prove that $K$ is continuous. Let $v_{n} \in P C(J)$ and $v_{n} \rightarrow v$ in $P C(J)$. It is easy to see that

$$
\int_{0}^{T}\left|\left(K v_{n}(t)-K v(t)\right)^{\prime}\right|^{2} d t=\int_{0}^{T}\left|G v_{n}(t)-G v(t)\right|^{2} d t \leq L^{2} \int_{0}^{T}\left|v_{n}(t)-v(t)\right|^{2} d t
$$

Therefore $\left(\int_{0}^{T}\left|\left(K v_{n}(t)-K v(t)\right)^{\prime}\right|^{2} d t\right)^{1 / 2} \leq \sqrt{T} L\left\|v_{n}-v\right\|_{P C(J)} \rightarrow 0$ as $n \rightarrow \infty$. 
Note that $\Delta\left(K v_{n}-K v\right)\left(t_{k}\right)=I_{k}\left(v_{n}\left(t_{k}\right)\right)-I_{k}\left(v\left(t_{k}\right)\right)$, and we have

$$
\begin{aligned}
K v_{n}(t)-K v(t)= & -\frac{1}{2}\left[\sum_{i=1}^{p}\left(I_{i}\left(v_{n}\left(t_{i}\right)\right)-I_{i}\left(v\left(t_{i}\right)\right)\right)+\int_{0}^{T}\left(K v_{n}-K v\right)^{\prime}(s) d s\right] \\
& +\int_{0}^{t}\left(K v_{n}-K v\right)^{\prime}(s) d s, \quad \text { for } t \in\left[0, t_{1}\right), \\
K v_{n}(t)-K v(t)= & -\frac{1}{2}\left[\sum_{i=1}^{p}\left(I_{i}\left(v_{n}\left(t_{i}\right)\right)-I_{i}\left(v\left(t_{i}\right)\right)\right)+\int_{0}^{T}\left(K v_{n}-K v\right)^{\prime}(s) d s\right] \\
& +\sum_{i=1}^{k}\left(I_{i}\left(v_{n}\left(t_{i}\right)\right)-I_{i}\left(v\left(t_{i}\right)\right)\right)+\int_{0}^{t}\left(K v_{n}-K v\right)^{\prime}(s) d s
\end{aligned}
$$

for $t \in\left[t_{k}, t_{k+1}\right), k=1,2, \ldots, p$. From the continuity of $I_{i}, i=1,2, \ldots, p$, and $\int_{0}^{T} \mid\left(K v_{n}(t)-\right.$ $K v(t))\left.^{\prime}\right|^{2} d t \rightarrow 0$ as $n \rightarrow \infty$, we deduce that $K$ is continuous.

For each $v \in P C(J)$, notice that $0=G 0$, so we have

$$
\left(\int_{0}^{T}|K v|^{2} d t\right)^{1 / 2} \leq \sqrt{T} L\|v\|_{P C(J)}+\left(\int_{0}^{T}|f(s)|^{2} d s\right)^{1 / 2}
$$

From (3.9) and Lemma 3.1, we know that $K$ maps bounded subsets of $P C(J)$ to relatively compact subsets of $P C(J)$.

Finally, for $\forall \lambda \in(0,1]$, we prove that the set of solutions of $u=\lambda K u$ is bounded. If $u=\lambda K u$ for some $\lambda \in(0,1)$, then

$$
\begin{gathered}
u^{\prime}(t)=\lambda G u(t)+\lambda f(t) \quad \text { a.e. } t \in J=[0, T], t \neq t_{k}, \\
u(0)=-u(T), \\
\Delta u\left(t_{k}\right)=\lambda I_{k}\left(u\left(t_{k}\right)\right), \quad k=1,2, \ldots, p .
\end{gathered}
$$

Therefore we have

$$
u(t)=-\frac{1}{2} \lambda\left[\Sigma_{i=1}^{p} I_{i}\left(u_{i}\left(t_{i}\right)\right)+\int_{0}^{T}(G u(s)+f(s)) d s\right]+\lambda \int_{0}^{t}(G(u(s))+f(s)) d s
$$

for $t \in\left[0, t_{1}\right)$, and

$$
\begin{aligned}
u(t)= & -\frac{1}{2} \lambda\left[\Sigma_{i=1}^{p} I_{i}\left(u_{i}\left(t_{i}\right)\right)+\int_{0}^{T}(G u(s)+f(s)) d s\right]+\lambda \sum_{i=1}^{k} I_{i}\left(u_{i}\left(t_{i}\right)\right) \\
& +\lambda \int_{0}^{t}(G(u(s))+f(s)) d s
\end{aligned}
$$


for $t \in\left(t_{k}, t_{k+1}\right], k=1, \ldots, p$. This implies that

$$
\|u\|_{P C(J)} \leq \frac{1}{2}\left[\gamma\|u\|_{P C(J)}+\delta+\int_{0}^{T}(|G u(s)|+|f(s)|) d s\right] .
$$

Since $0=G 0$, and $|G u| \leq L|u|$, so we have

$$
\|u\|_{P C(J)} \leq \frac{1}{2}\left[1-\frac{1}{2}(\gamma+T L)\right]^{-1}\left(\delta+\int_{0}^{T}|f(s)| d s\right) .
$$

The Leray-Schauder principle guarantees a fixed point of $K$, which is easily seen to be a solution of (E 3.2).

By using a similar method to Theorem 3.2, one can deduce the following result.

Theorem 3.3. Let $G: R^{n} \rightarrow R^{n}$ be a function satisfying $G 0=0$, and $f(t, x):[0, T] \times R^{n} \rightarrow R^{n}$ a Caratheodory function, that is, $f$ is measurable in $t$ for each $x \in R^{n}$, and $f$ is continuous in $x$ for each $t \in[0, T]$, such that $|f(t, x)| \leq g(t)$ for $(t, x) \in[0, T] \times R^{n}$, where $g(\cdot) \in L^{2}([0, T])$, and let $I_{k}: R^{n} \rightarrow R^{n}$ be continuous functions for $k=1,2, \ldots, p$. Suppose the following conditions are satisfied:

(1) $|G u-G v| \leq L|u-v|$ for all $u, v \in R^{n}$, and $L>0$ is a constant;

(2) $\Sigma_{k=1}^{p}\left|I_{k}\left(x_{k}\right)\right| \leq \gamma\left\{\max _{1 \leq k \leq p}\left|x_{k}\right|\right\}+\delta$ for all $x_{k} \in R^{n}, k=1,2, \ldots, p$, where $\gamma, \delta>0$ are constants;

(3) $\gamma+T L<2$.

Then the equation

$$
\begin{gathered}
u^{\prime}(t)=G u(t)+f(t, u(t)), \quad \text { a.e. } t \in J=[0, T], t \neq t_{k}, \\
u(0)=-u(T), \\
\Delta u\left(t_{k}\right)=I_{k}\left(u\left(t_{k}\right)\right), \quad k=1,2, \ldots, p
\end{gathered}
$$

has a solution.

\section{Examples}

In this section, we give examples to show the application of our results to differential and impulsive differential equations. 
Example 4.1. Consider the antiperiodic problem

$$
\begin{gathered}
u_{1}^{\prime}(t)=\lambda_{1} u_{1}(t)+5 u_{2}(t)+\sin \pi t, \quad t \in R, \\
u_{2}^{\prime}(t)=\frac{7}{2} u_{1}(t)+\lambda_{2} u_{2}(t)+\cos \pi t, \quad t \in R, \\
u_{1}(t)=-u_{1}(t+1), \quad u_{2}(t)=-u_{2}(t+1), \quad t \in R .
\end{gathered}
$$

Set

$$
u=\left(\begin{array}{l}
u_{1} \\
u_{2}
\end{array}\right), \quad f(t)=\left(\begin{array}{c}
\sin \pi t \\
\cos \pi t
\end{array}\right), \quad A=\left(\begin{array}{cc}
\lambda_{1} & 5 \\
7 & \lambda_{2}
\end{array}\right)
$$

Now (E 4.1) is equivalent to

$$
\begin{gathered}
u^{\prime}(t)=A u(t)+f(t), \quad t \in R, \\
u(t)=-u(t+1), \quad t \in R .
\end{gathered}
$$

Also $f(t)=-f(t+1)$, for $t \in R$ and $(1 / 2)\left|a_{12}-a_{21}\right|=3 / 4$. By Theorem 2.1, (E 4.2) has a unique solution, so (E 4.1) has a unique solution.

Example 4.2. Consider the antiperiodic boundary value problem

$$
\begin{gathered}
u_{1}^{\prime}(t)=\frac{1}{2+u_{1}^{2}(t)+u_{2}^{2}(t)}\left[3 u_{1}(t)-2 u_{2}(t)\right]+\sin \pi t, \quad t \in(0,1), t \neq \frac{1}{4} \\
u_{2}^{\prime}(t)=\frac{1}{2+u_{1}^{2}(t)+u_{2}^{2}(t)}\left[2 u_{1}(t)+3 u_{2}(t)\right]-\cos \pi t, \quad t \in(0,1), t \neq \frac{1}{4} \\
\Delta u_{1}\left(\frac{1}{4}\right)=\frac{1}{5\left(1+\left|u_{2}(1 / 4)\right|\right)^{\prime}}, \quad \Delta u_{2}\left(\frac{1}{4}\right)=\frac{1}{8\left(1+\left|u_{1}(1 / 4)\right|\right)} \\
u_{1}(0)=-u_{1}(1), \quad u_{2}(0)=-u_{2}(1) .
\end{gathered}
$$

Set

$$
u=\left(\begin{array}{l}
u_{1} \\
u_{2}
\end{array}\right), \quad f(t)=\left(\begin{array}{c}
\sin \pi t \\
-\cos \pi t
\end{array}\right), \quad G u=\left(\begin{array}{c}
\frac{3 u_{1}-2 u_{2}}{2+u_{1}^{2}+u_{2}^{2}} \\
\frac{2 u_{1}+3 u_{2}}{2+u_{1}^{2}+u_{2}^{2}}
\end{array}\right), \quad I(u)\left(\begin{array}{c}
\frac{1}{5\left(1+\left|u_{2}\right|\right)} \\
\frac{1}{8\left(1+\left|u_{1}\right|\right)}
\end{array}\right) .
$$


It is easy to check that $|G u-G v| \leq(\sqrt{13} / 2)|u-v|$ for $u, v \in R^{2},|I(u)|<2 / 5$ for $u \in R^{2}$, and $\sqrt{13} / 2<2$. Now (E 4.3) is equivalent to the equation

$$
\begin{gathered}
u^{\prime}(t)=G u(t)+f(t), \quad t \in(0,1), t \neq \frac{1}{4} \\
\Delta u\left(\frac{1}{4}\right)=I\left(u\left(\frac{1}{4}\right)\right), \quad u(0)=-u(1) .
\end{gathered}
$$

By Theorem 3.2, we know that (E 4.4) has a solution, so (E 4.3) has a solution.

\section{Acknowledgment}

The first author is supported by an NSFC Grant, Grant no. 10871052.

\section{References}

[1] H. Okochi, "On the existence of periodic solutions to nonlinear abstract parabolic equations," Journal of the Mathematical Society of Japan, vol. 40, no. 3, pp. 541-553, 1988.

[2] A. R. Aftabizadeh, S. Aizicovici, and N. H. Pavel, "On a class of second-order anti-periodic boundary value problems," Journal of Mathematical Analysis and Applications, vol. 171, no. 2, pp. 301-320, 1992.

[3] A. R. Aftabizadeh, S. Aizicovici, and N. H. Pavel, "Anti-periodic boundary value problems for higher order differential equations in Hilbert spaces," Nonlinear Analysis: Theory, Methods E Applications, vol. 18, no. 3, pp. 253-267, 1992.

[4] B. Ahmad and V. Otero-Espinar, "Existence of solutions for fractional differential inclusions with antiperiodic boundary conditions," Boundary Value Problems, vol. 2009, Article ID 625347, 11 pages, 2009.

[5] B. Ahmad and J. J. Nieto, "Existence and approximation of solutions for a class of nonlinear impulsive functional differential equations with anti-periodic boundary conditions," Nonlinear Analysis: Theory, Methods \& Applications, vol. 69, no. 10, pp. 3291-3298, 2008.

[6] S. Aizicovici and N. H. Pavel, "Anti-periodic solutions to a class of nonlinear differential equations in Hilbert space," Journal of Functional Analysis, vol. 99, no. 2, pp. 387-408, 1991.

[7] A. Cabada and D. R. Vivero, "Existence and uniqueness of solutions of higher-order antiperiodic dynamic equations," Advances in Difference Equations, vol. 2004, no. 4, pp. 291-310, 2004.

[8] Y. Q. Chen, "Note on Massera's theorem on anti-periodic solution," Advances in Mathematical Sciences and Applications, vol. 9, pp. 125-128, 1999.

[9] Y. Q. Chen, X. Wang, and H. Xu, "Anti-periodic solutions for semilinear evolution equations," Journal of Mathematical Analysis and Applications, vol. 273, no. 2, pp. 627-636, 2002.

[10] Y. Q. Chen, Y. J. Cho, and J. S. Jung, "Antiperiodic solutions for semilinear evolution equations," Mathematical and Computer Modelling, vol. 40, no. 9-10, pp. 1123-1130, 2004.

[11] Y. Q. Chen, Y. J. Cho, and D. O'Regan, “Anti-periodic solutions for evolution equations with mappings in the class $\left(\mathrm{S}_{+}\right)$," Mathematische Nachrichten, vol. 278, no. 4, pp. 356-362, 2005.

[12] Y. Q. Chen, "Anti-periodic solutions for semilinear evolution equations," Journal of Mathematical Analysis and Applications, vol. 315, no. 1, pp. 337-348, 2006.

[13] Y. Q. Chen, Y. J. Cho, and F. L. Wang, "Anti-periodic boundary value problems for impulsive differential equations," International Journal of Computational and Applied Mathematics, vol. 1, pp. 9$16,2006$.

[14] Y. Q. Chen, J. J. Nieto, and D. O’Regan, “Anti-periodic solutions for fully nonlinear first-order differential equations," Mathematical and Computer Modelling, vol. 46, no. 9-10, pp. 1183-1190, 2007.

[15] D. Franco and J. J. Nieto, "First-order impulsive ordinary differential equations with anti-periodic and nonlinear boundary conditions," Nonlinear Analysis: Theory, Methods E Applications, vol. 42, no. 2, pp. 163-173, 2000.

[16] D. Franco, J. J. Nieto, and D. O’Regan, "Anti-periodic boundary value problem for nonlinear first order ordinary differential equations," Mathematical Inequalities and Applications, vol. 6, no. 3, pp. 477485, 2003. 
[17] D. Franco, J. J. Nieto, and D. O'Regan, "Existence of solutions for first order ordinary differential equations with nonlinear boundary conditions," Applied Mathematics and Computation, vol. 153, no. 3, pp. 793-802, 2004.

[18] A. Haraux, "Anti-periodic solutions of some nonlinear evolution equations," Manuscripta Mathematica, vol. 63, no. 4, pp. 479-505, 1989.

[19] Y. Li and L. Huang, "Anti-periodic solutions for a class of Liénard-type systems with continuously distributed delays," Nonlinear Analysis: Real World Applications, vol. 10, no. 1, pp. 2127-2132, 2009.

[20] B. Liu, "An anti-periodic LaSalle oscillation theorem for a class of functional differential equations," Journal of Computational and Applied Mathematics, vol. 223, no. 2, pp. 1081-1086, 2009.

[21] B. Liu, "Anti-periodic solutions for forced Rayleigh-type equations," Nonlinear Analysis: Real World Applications, vol. 10, no. 5, pp. 2850-2856, 2009.

[22] Z. Luo, J. Shen, and J. J. Nieto, "Antiperiodic boundary value problem for first-order impulsive ordinary differential equations," Computers and Mathematics with Applications, vol. 49, no. 2-3, pp. 253261, 2005.

[23] M. Nakao, "Existence of an anti-periodic solution for the quasilinear wave equation with viscosity," Journal of Mathematical Analysis and Applications, vol. 204, no. 3, pp. 754-764, 1996.

[24] H. Okochi, "On the existence of anti-periodic solutions to a nonlinear evolution equation associated with odd subdifferential operators," Journal of Functional Analysis, vol. 91, no. 2, pp. 246-258, 1990.

[25] H. Okochi, "On the existence of anti-periodic solutions to nonlinear parabolic equations in noncylindrical domains," Nonlinear Analysis: Theory, Methods E Applications, vol. 14, no. 9, pp. 771783, 1990.

[26] P. Souplet, "Uniqueness and nonuniqueness results for the antiperiodic solutions of some secondorder nonlinear evolution equations," Nonlinear Analysis: Theory, Methods \& Applications, vol. 26, no. 9, pp. 1511-1525, 1996.

[27] P. Souplet, "Optimal uniqueness condition for the antiperiodic solutions of some nonlinear parabolic equations," Nonlinear Analysis: Theory, Methods E Applications, vol. 32, no. 2, pp. 279-286, 1998.

[28] K. Wang, "A new existence result for nonlinear first-order anti-periodic boundary value problems," Applied Mathematics Letters, vol. 21, no. 11, pp. 1149-1154, 2008.

[29] K. Wang and Y. Li, "A note on existence of (anti-)periodic and heteroclinic solutions for a class of second-order odes," Nonlinear Analysis: Theory, Methods \& Applications, vol. 70, no. 4, pp. 1711-1724, 2009.

[30] R. Wu, "An anti-periodic LaSalle oscillation theorem," Applied Mathematics Letters, vol. 21, no. 9, pp. 928-933, 2008.

[31] Y. Yin, "Monotone iterative technique and quasilinearization for some anti-periodic problem," Nonlinear World, vol. 3, pp. 253-266, 1996.

[32] F.-J. Delvos and L. Knoche, "Lacunary interpolation by antiperiodic trigonometric polynomials," BIT Numerical Mathematics, vol. 39, no. 3, pp. 439-450, 1999.

[33] J. Du, H. Han, and G. Jin, "On trigonometric and paratrigonometric Hermite interpolation," Journal of Approximation Theory, vol. 131, no. 1, pp. 74-99, 2004.

[34] H. L. Chen, "Antiperiodic wavelets," Journal of Computational Mathematics, vol. 14, no. 1, pp. 32-39, 1996.

[35] A. Abdurrahman, F. Anton, and J. Bordes, "Half-string oscillator approach to string field theory (ghost sector I)," Nuclear Physics B, vol. 397, no. 1-2, pp. 260-282, 1993.

[36] C. Ahn and C. Rim, "Boundary flows in general coset theories," Journal of Physics A, vol. 32, no. 13, pp. 2509-2525, 1999.

[37] Y. Li and L. Yang, "Anti-periodic solutions for Cohen-Grossberg neural networks with bounded and unbounded delays," Communications in Nonlinear Science and Numerical Simulation, vol. 14, no. 7, pp. 3134-3140, 2009.

[38] M. Pawellek, "Quantization of sine-Gordon solitons on the circle: semiclassical vs. exact results," Nuclear Physics B, vol. 810, no. 3, pp. 527-541, 2009.

[39] S. Pinsky and U. Trittmann, "Antiperiodic boundary conditions in supersymmetric discrete light cone quantization," Physical Review D, vol. 62, no. 8, Article ID 087701, 4 pages, 2000.

[40] J. Shao, "Anti-periodic solutions for shunting inhibitory cellular neural networks with time-varying delays," Physics Letters A, vol. 372, no. 30, pp. 5011-5016, 2008. 